\title{
Water Uptake and Loss in Viscous Aerosol Particles with Concentration-Dependent Diffusivities
}

\author{
April 30, 2019 \\ Brandon J. Wallace and Thomas C. Preston* \\ Department of Atmospheric and Oceanic Sciences \\ and Department of Chemistry, \\ McGill University, 805 Sherbrooke Street West, Montreal, QC, Canada H3A 0B9
}

published in the Journal of Physical Chemistry A 2019, 123, 3374-3382

8 Figures and 24 manuscript pages

${ }^{*}$ Thomas C. Preston
e-mail: thomas.preston@mcgill.ca 


\section{Abstract}

An accurate understanding of the equilibration timescale of organic aerosol particles with surrounding water vapour is difficult due to the strong concentration-dependent diffusivities that are present in these systems. We examine this problem along with the closely related problem of the time-dependent radius of a binary aerosol particle during the uptake or loss of water. The governing equations and boundary conditions are discussed and a boundary value problem is formulated and solved. The resulting expressions are applied to water uptake and loss in two systems of atmospheric importance: aqueous-inorganic particles and high viscosity organic particles. Accuracy is evaluated through a comparison with numerical solutions. For particles whose diffusivity has a strong dependence on water concentration and whose viscosity remains above $1 \mathrm{~Pa} \cdot \mathrm{s}$ during water uptake or loss, the expression for the characteristic equilibration time is found to be in excellent agreement with numerical results. Moreover, it provides physical insight into the mass transport processes. 


\section{Introduction}

The treatment of the time-dependent radius of a single component droplet using Maxwell's description of the condensational growth and evaporation and quasi-stationary analysis has been found to be accurate for most cases of meteorological interest. ${ }^{1}$ Consideration of multicomponent particles introduces additional complexities to the analysis and a common simplification is that the composition of the condensed phase is uniform (the particle is spatially homogeneous during evaporation or condensation). ${ }^{2,3}$ However, this assumption will not be satisfactory when condensed phase diffusion determines the equilibration timescale for gas-particle partitioning. ${ }^{4}$ In the atmosphere, one well-studied example of this type of behaviour is the uptake and loss of water by high viscosity secondary organic aerosol particles. ${ }^{5-11}$

As uptake or loss of volatile species occur, the density profile inside the particle changes and particle growth or shrinkage takes place. For a spherical particle, numerical methods have been used to calculate the resulting time-dependent concentration profile and radius. ${ }^{12-15}$ In these methods, space is discretized by dividing the particle into concentric shells. As mass transport takes place, the volume of each shell is adjusted and the overall particle radius can be determined by simply adding up all of the resulting shell thicknesses after each time step. ${ }^{14}$

Due to the physical accuracy of these numerical methods it is somewhat surprising to realize that the underlying models are not amenable to an analytic solution. There are two reasons for this. First, the appropriate condition for the conservation of mass at a moving boundary is absent from these models. Second, because of the way diffusion and density changes are treated, the convection-diffusion and continuity equations are not directly used when calculating mass transport. In Section 2, we formulate the boundary value problem using the moving boundary 
condition and we use the convection-diffusion equation and the continuity equation as the governing equations. In Section 3, using several approximations, we solve the boundary value problem within the continuum regime under isothermal conditions and determine an expression for the time-dependent radius of a spherical particle containing two species, one volatile and one non-volatile. The case that is considered is one where the vapour phase concentration of the volatile species is instantaneously changed. We restrict our attention to the case where the volatile species is water. Using the derived expression, in Section 4, the time-dependent radius during the uptake and loss of water by aqueous-inorganic and high viscosity organic particles are calculated and these results are compared to numerical calculations.

Understanding how condensed phase diffusion controls water uptake and loss in secondary organic aerosol has important implications for atmospheric particles in terms of growth, phase, optical properties, and nuclei activity. ${ }^{6,9,16-21}$ In Section 5, we consider the characteristic equilibration time of high viscosity aerosol particles with the surrounding relative humidity $(\mathrm{RH})$. This problem is closely connected to that of the time-dependent particle radius that is discussed in the earlier sections. To our knowledge, aside from expressions that provide limits on the equilibration time,$^{9}$ currently only numerical methods allow for the determination of this characteristic time. We derive an expression for the equilibration time and assess its accuracy using the three model organic systems with high viscosity. 


\section{The Uptake and Loss of Water from a Binary Aerosol Particle}

For a spherical particle with a time-dependent radius $a(t)$, composed of water, w, and a nonvolatile solute, s, with mass concentrations $\rho_{\mathrm{w}}$ and $\rho_{\mathrm{s}}$, mass fractions $w_{\mathrm{w}}$ and $w_{\mathrm{s}}$, and velocities $\mathbf{v}_{\mathrm{w}}$ and $\mathbf{v}_{\mathrm{s}}$, the mass density of the solution will be $\rho=\rho_{\mathrm{w}}+\rho_{\mathrm{s}}$ and the mass average velocity will be $\mathbf{v}=w_{\mathrm{w}} \mathbf{v}_{\mathrm{w}}+w_{\mathrm{s}} \mathbf{v}_{\mathrm{s}}$. We will assume that the velocity field is entirely radial so its components are $v_{r}=v_{r}(r, t)$ and $v_{\theta}=v_{\phi}=0$. Then, inside the particle, the convection-diffusion equation for water will be

$$
\frac{\partial \rho_{\mathrm{w}}}{\partial t}+\frac{1}{r^{2}} \frac{\partial}{\partial r}\left(r^{2} \rho_{\mathrm{w}} v_{r}\right)=\frac{1}{r^{2}} \frac{\partial}{\partial r}\left(r^{2} \rho D \frac{\partial w_{\mathrm{w}}}{\partial r}\right)
$$

where $D$ is the concentration-dependent diffusivity, and the continuity equation will be

$$
\frac{\partial \rho}{\partial t}+\frac{1}{r^{2}} \frac{\partial}{\partial r}\left(r^{2} \rho v_{r}\right)=0
$$

We will assume that mass transport in the vapour phase is governed by the steady-state equation

$$
\frac{\partial}{\partial r}\left(r^{2} \frac{\partial \rho_{\mathrm{w}}^{\mathrm{v}}}{\partial r}\right)=0 \text { for } a(t)<r<\infty,
$$

where $\rho_{\mathrm{w}}^{\mathrm{v}}$ is the vapour phase mass concentration of water.

If the time-dependent vapour phase concentration of water at $r=a(t)$ is $\rho_{\mathrm{w}_{a}}^{\mathrm{v}}(t)$ and the concentration of water at $r=\infty$ is $\rho_{\mathrm{w}_{f}}^{\mathrm{v}}$, the solution to Eq. 3 is

$$
\rho_{\mathrm{w}}^{\mathrm{v}}(r, t)=\rho_{\mathrm{w}_{f}}^{\mathrm{v}}+\frac{a(t)}{r}\left(\rho_{\mathrm{w}_{a}}^{\mathrm{v}}(t)-\rho_{\mathrm{w}_{f}}^{\mathrm{v}}\right)
$$

At the centre of the spherical particle, the boundary conditions from symmetry are

$$
\frac{\partial \rho_{\mathrm{w}}}{\partial r}=0, \quad \frac{\partial \rho}{\partial r}=0, \quad \text { and } \quad v_{r}=0 \quad \text { at } \quad r=0
$$


Conservation of mass for water at the particle surface gives the boundary condition

$$
\left(\rho_{\mathrm{w}}^{\mathrm{v}}-\rho_{\mathrm{w}}\right) \frac{d a}{d t}=D \rho \frac{\partial w_{\mathrm{w}}}{\partial r}-D_{\mathrm{v}} \frac{\partial \rho_{\mathrm{w}}^{\mathrm{v}}}{\partial r}-v_{r} \rho_{\mathrm{w}} \quad \text { at } \quad r=a(t)
$$

where $D_{\mathrm{v}}$ is the diffusivity of water in the vapour phase. In Eq. 6 we have assumed that the mass average velocity in the vapour phase is zero and the mass density of the vapour phase is constant. These two assumptions are consistent with Eq. 3.

When the solute is non-volatile, conservation of mass yields the boundary condition ${ }^{22,23}$

$$
\frac{d a}{d t}=v_{r}+\frac{D}{1-w_{\mathrm{w}}} \frac{\partial w_{\mathrm{w}}}{\partial r} \quad \text { at } \quad r=a(t)
$$

Initially, the mass concentration of water inside the particle will be uniform and set to a constant value of $\rho_{\mathrm{w}_{i}}$ and the mass average velocity will be zero. Therefore,

$$
\rho_{\mathrm{w}}=\rho_{\mathrm{w}_{i}} \quad \text { and } \quad v_{r}=0 \quad \text { for } \quad 0<r<a_{0}, \quad t=0
$$

where $a_{0}$ is the initial radius of the spherical particle.

We are interested in a model that describes how $a(t)$ changes in response to a stepwise change in the vapour phase concentration of water. For $t>0$, the vapour phase concentration of water at $r=\infty$ is changed from its initial value to its final value, $\rho_{\mathrm{w}_{f}}^{\mathrm{v}}$. As $t \rightarrow \infty$, the concentration of water inside the particle will approach a uniform distribution with a constant value of $\rho_{\mathrm{w}_{f}}$. To proceed, we assume that between $\rho_{\mathrm{w}_{i}}$ and $\rho_{\mathrm{w}_{f}}$, the mass density can be accurately expressed as a linear function of $\rho_{\mathrm{w}}:{ }^{24}$

$$
\rho=\rho_{m}+\alpha\left(\rho_{\mathrm{w}}-\rho_{\mathrm{w}_{m}}\right)
$$

where

$$
\rho_{\mathrm{w}_{m}}=\frac{\rho_{\mathrm{w}_{i}}+\rho_{\mathrm{w}_{f}}}{2}, \quad \rho_{m}=\rho\left(\rho_{\mathrm{w}_{m}}\right), \quad \text { and } \quad \alpha=\left.\frac{d \rho}{d \rho_{\mathrm{w}}}\right|_{\rho_{\mathrm{w}}=\rho_{\mathrm{w}_{m}}}
$$


Inserting Eq. 9 into the partial time derivative in Eq. 2 and combining the result with Eq. 1 we find

$$
\frac{1}{r^{2}} \frac{\partial}{\partial r}\left(r^{2} \rho_{\mathrm{w}} v_{r}\right)-\frac{1}{\alpha r^{2}} \frac{\partial}{\partial r}\left(r^{2} \rho v_{r}\right)=\frac{1}{r^{2}} \frac{\partial}{\partial r}\left(r^{2} \rho D \frac{\partial w_{\mathrm{w}}}{\partial r}\right) .
$$

Integrating Eq. 10 from 0 to $r$ and applying the boundary conditions from Eq. 5 yields the following expression for $v_{r}$ :

$$
v_{r}=\frac{\alpha \rho D}{\alpha \rho_{\mathrm{w}}-\rho} \frac{\partial w_{\mathrm{w}}}{\partial r}
$$

Inserting Eq. 11 into Eq. 1 gives

$$
\frac{\partial \rho_{\mathrm{w}}}{\partial t}=\frac{1}{r^{2}} \frac{\partial}{\partial r}\left(r^{2} \rho D_{c} \frac{\partial w_{\mathrm{w}}}{\partial r}\right)
$$

where $D_{c}=D\left(1-\alpha \rho_{\mathrm{w}} /\left(\alpha \rho_{\mathrm{w}}-\rho\right)\right)$.

Eq. 12 is then integrated from $r=0$ to $r=a(t)$ to obtain

$$
\frac{d}{d t}\left(\int_{0}^{a(t)} r^{2} \rho_{\mathrm{w}} d r\right)=\left.a^{2}\left(\rho_{\mathrm{w}} \frac{d a}{d t}+\rho D_{c} \frac{\partial w_{\mathrm{w}}}{\partial r}\right)\right|_{r=a(t)} .
$$

Inserting Eq. 11 into Eq. 7 and applying the result to Eq. 13 yields

$$
\frac{d}{d t}\left(\int_{0}^{a(t)} r^{2} \rho_{\mathrm{w}} d r\right)=\epsilon a^{2} \frac{d a}{d t}
$$

where $\epsilon=\left(\alpha \rho_{\mathrm{w}_{m}}-\rho_{m}\right) /(\alpha-1)$.

Integrating Eq. 14 from 0 to $t$ one obtains the integral equation

$$
\epsilon a(t)^{3}=3 \int_{0}^{a(t)} r^{2} \rho_{\mathrm{w}} d r+a_{0}^{3}\left(\epsilon-\rho_{\mathrm{w}_{i}}\right)
$$

It can readily be seen that when $\rho_{\mathrm{w}}=\rho_{\mathrm{w}_{i}}$, Eq. 15 gives the expected result of $a=a_{0}$. Also, when $t \rightarrow \infty, \rho_{\mathrm{w}}=\rho_{\mathrm{w}_{f}}$ and $a(t)$ approaches its final value

$$
a_{\infty}=a_{0}\left(\frac{\epsilon-\rho_{\mathrm{w}_{i}}}{\epsilon-\rho_{\mathrm{w}_{f}}}\right)^{1 / 3}
$$


In single particle experiments, the total amount of water in a particle, $Q_{\mathrm{w}}$, can, in principle, be obtained by integrating the scattered Raman intensity of the water bands (although the presence of Mie resonances will complicate this type of analysis). ${ }^{25-27}$ The relationship between $\rho_{\mathrm{w}}$ and $Q_{\mathrm{w}}$ at any $t$ will be

$$
Q_{\mathrm{w}}(t)=4 \pi \int_{0}^{a(t)} r^{2} \rho_{\mathrm{w}} d r
$$

Inserting Eq. 17 into Eq. 15 gives us

$$
a(t)=\left(\frac{3}{4 \pi \epsilon} Q_{\mathrm{w}}(t)+a_{0}^{3}\left(1-\frac{\rho_{\mathrm{w}_{i}}}{\epsilon}\right)\right)^{1 / 3} .
$$

Eq. 18 provides an experimentally accessible way to track $a(t)$ as it does not require knowledge of $\rho_{\mathrm{w}}(r, t)$ but only the potentially measurable quantity $Q_{\mathrm{w}}(t)$. When deriving Eq. 18, no assumptions concerning the concentration profile inside the particle were made nor was it ever necessary to assume that $D$ was constant. Although it is beyond the scope of this work, if a particle is large enough that $a(t)$ can be tracked using optical microscopy then $Q_{\mathrm{w}}(t)$ can readily be calculated from Eq. 18 using such measurements.

While $a(t)$ can be obtained without knowing the time-dependent concentration profile of water, the same is not true for D. Inserting Eq. 11 into Eq. 7 one finds that the relationship between $a(t)$ and $D$ is

$$
\frac{d a}{d t}=-\frac{\rho D}{\epsilon\left(w_{\mathrm{w}}-1\right)} \frac{\partial w_{\mathrm{w}}}{\partial r}
$$

Therefore, in addition to $a(t)$, knowledge of both the time-dependent $w_{\mathrm{w}}$ and its gradient at $r=a(t)$ is required to determine $D$. 


\section{An Expression for the Time-dependent Radius of a Binary Aerosol Particle}

To determine $\rho_{\mathrm{w}}(r, t)$ for Eq. 15 that is suitable across all $t$ we will formulate a linear boundary value problem where we will assume that $a(t)$ is stationary and fixed at $a_{0}$. With this consideration in mind, we set $D_{c}=\bar{D}$ in Eq. 12 , where $\bar{D}$ is a constant to be subsequently determined. This gives the linear partial differential equation:

$$
\frac{\partial \rho_{\mathrm{w}}}{\partial t}=\frac{\bar{D}}{r^{2}} \frac{\partial}{\partial r}\left(r^{2} \frac{\partial \rho_{\mathrm{w}}}{\partial r}\right) \quad \text { for } \quad 0<r<a_{0}
$$

where the boundary condition at the centre of the sphere is

$$
\frac{\partial \rho_{\mathrm{w}}}{\partial r}=0 \quad \text { at } \quad r=0
$$

and, for the assumption that the radius of the particle is not changing with time, conservation of mass of water gives the boundary condition

$$
\bar{D} \frac{\partial \rho_{\mathrm{w}}}{\partial r}=D_{\mathrm{v}} \frac{\partial \rho_{\mathrm{w}}^{\mathrm{v}}}{\partial r} \quad \text { at } \quad r=a_{0}
$$

Finally, the initial condition for this problem is

$$
\rho_{\mathrm{w}}=\rho_{\mathrm{w}_{i}} \quad \text { for } \quad 0<r<a_{0}, \quad t=0 .
$$

Eq. 22 requires the concentration profile of water in the vapour phase and a relationship between $\rho_{\mathrm{w}}$ and $\rho_{\mathrm{w}}^{\mathrm{v}}$ at $r=a_{0}$. In order to describe the concentration profile in the vapour phase, we use Eq. 4 (again, setting $a(t)=a_{0}$ ). For the relationship between the liquid and vapour phase concentration of water at the particle surface, we ignore the effect of surface curvature and write the modified Raoult's law as

$$
\gamma x_{\mathrm{w}}=p_{\mathrm{w}} / p_{\mathrm{w}}^{\circ} \quad \text { at } \quad r=a_{0}
$$


where $x_{\mathrm{w}}$ is the mole fraction of water, $\gamma$ is the activity coefficient of water for $x_{\mathrm{w}}, p_{\mathrm{w}}$ is the partial pressure of water at the surface, and $p_{\mathrm{w}}^{\circ}$ is the saturation vapour pressure of water.

When expressed in terms of mass concentrations, Eq. 24 becomes

$$
\gamma \frac{\rho_{\mathrm{w}} / M_{\mathrm{w}}}{\rho_{\mathrm{w}} / M_{\mathrm{w}}+\rho_{\mathrm{s}} / M_{\mathrm{s}}}=\frac{\rho_{\mathrm{w}}^{\mathrm{v}} R T}{M_{\mathrm{w}} p_{\mathrm{w}}^{\circ}} \quad \text { at } \quad r=a_{0}
$$

where $M_{\mathrm{w}}$ and $M_{\mathrm{s}}$ are the molar masses of water and the solute, respectively, $R$ is the gas constant, and $T$ is the temperature. If Eq. 25 is combined with Eq. 4 and the result is inserted into Eq. 22 then a nonlinear boundary condition will be obtained. To avoid this, an approximate form of Eq. 25 is used here instead. This form is found by expanding the left-hand side of Eq. 25 using a first order power series about the point $\rho_{\mathrm{w}}=\rho_{\mathrm{w}_{f}}$ to yield

$$
\rho_{\mathrm{w}}^{\mathrm{v}}=k \rho_{\mathrm{w}}+q \quad \text { at } \quad r=a_{0},
$$

where

$$
\begin{aligned}
& k=\frac{\gamma_{f} M_{\mathrm{w}}^{2} M_{\mathrm{s}} p_{\mathrm{w}}^{\circ}\left(\rho_{m}-\alpha \rho_{\mathrm{w}_{m}}\right)}{R T\left(M_{\mathrm{w}}\left((\alpha-1) \rho_{\mathrm{w}_{f}}-\alpha \rho_{\mathrm{w}_{m}}+\rho_{m}\right)+M_{\mathrm{s}} \rho_{\mathrm{w}_{f}}\right)^{2}}, \\
& q=\frac{\gamma_{f} M_{\mathrm{w}} M_{\mathrm{s}} p_{\mathrm{w}}^{\circ} \rho_{\mathrm{w}_{f}}^{2}\left((\alpha-1) M_{\mathrm{w}}+M_{\mathrm{s}}\right)}{R T\left(M_{\mathrm{w}}\left((\alpha-1) \rho_{\mathrm{w}_{f}}-\alpha \rho_{\mathrm{w}_{m}}+\rho_{m}\right)+M_{\mathrm{s}} \rho_{\mathrm{w}_{f}}\right)^{2}},
\end{aligned}
$$

and $\gamma_{f}$ is the activity coefficient of water when $\rho_{\mathrm{w}}=\rho_{\mathrm{w}_{f}}$. The point $\rho_{\mathrm{w}}=\rho_{\mathrm{w}_{f}}$ was chosen for the expansion in order to ensure that $a(t \rightarrow \infty)=a_{\infty}$.

Combining Eqs. 4, 22, and 26 results in the linear boundary condition

$$
\bar{D} \frac{\partial \rho_{\mathrm{w}}}{\partial r}=-\frac{k D_{\mathrm{v}}}{a_{0}}\left(\rho_{\mathrm{w}}-\rho_{\mathrm{w}_{f}}\right) \quad \text { at } \quad r=a_{0}
$$

The solution to the boundary value problem defined by Eqs. 20, 21, 23, and 27 is

$$
\rho_{\mathrm{w}}(r, t)=\rho_{\mathrm{w}_{f}}-\frac{2 a_{0} \vartheta}{r}\left(\rho_{\mathrm{w}_{f}}-\rho_{\mathrm{w}_{i}}\right) \sum_{n=1}^{\infty} B_{n} \sin \frac{\lambda_{n} r}{a_{0}} e^{-\bar{D} \lambda_{n}^{2} t / a_{0}^{2}}
$$


where

$$
B_{n}=\frac{\left(\lambda_{n}^{2}+\xi^{2}\right) \sin \lambda_{n}}{\lambda_{n}^{2}\left(\lambda_{n}^{2}+\xi(\xi-1)\right)}, \quad \lambda_{n} \cot \lambda_{n}=\xi, \quad \xi=1-\vartheta, \quad \text { and } \quad \vartheta=k D_{\mathrm{v}} / \bar{D}
$$

Inserting Eq. 28 into Eq. 15 yields the desired expression

$$
a(t)^{3}=\frac{\left(\rho_{\mathrm{w}_{i}}-\epsilon\right) a_{0}^{3}}{\rho_{\mathrm{w}_{f}}-\epsilon+6 \vartheta^{2}\left(\rho_{\mathrm{w}_{i}}-\rho_{\mathrm{w}_{f}}\right) \sum_{n=1}^{\infty}\left(B_{n} / \lambda_{n}^{2}\right) \sin \lambda_{n} e^{-\bar{D} \lambda_{n}^{2} t / a_{0}^{2}}} .
$$

Additionally, defining the dimensionless variables

$$
\tilde{a}=a(t) / a_{0}, \quad c_{i}=\rho_{\mathrm{w}_{i}} / \epsilon, \quad c_{f}=\rho_{\mathrm{w}_{f}} / \epsilon, \quad \text { and } \quad \tilde{t}=\bar{D} t / a_{0}^{2},
$$

allows Eq. 29 to be put in a dimensionless form

$$
\tilde{a}^{3}=\frac{c_{i}-1}{c_{f}-1+6 \vartheta^{2}\left(c_{i}-c_{f}\right) \sum_{n=1}^{\infty}\left(B_{n} / \lambda_{n}^{2}\right) \sin \lambda_{n} e^{-\lambda_{n}^{2} \tilde{t}}}
$$

Examination of Eq. 30 reveals that $\tilde{a}$ can be represented by a one-parameter family of curves. Fig. 1 shows $\tilde{a}$ as a function of $\tilde{t}$ for several values of $\vartheta$. As $\vartheta$ increases, the $\tilde{a}$ curves converge and become indistinguishable from each other (e.g. the curves for $\vartheta=1000$ and 10000 overlap). The physical situation for this large $\vartheta$ limit is one where the concentration at the surface of the particle is fixed at $c_{f}$ for all $\tilde{t}$ and transport of water is limited by diffusion inside the particle. This limiting behaviour can occur in the atmosphere during water uptake or loss by high viscosity aerosol particles. ${ }^{28}$ In Fig. 2 we use the dimensionless concentration

$$
c=c_{f}-\frac{2 \vartheta}{\tilde{r}}\left(c_{f}-c_{i}\right) \sum_{n=1}^{\infty} B_{n} \sin \lambda_{n} \tilde{r} e^{-\lambda_{n}^{2} \tilde{t}}
$$

where $c=\rho_{\mathrm{w}} / \epsilon$ and $\tilde{r}=r / a_{0}$, to illustrate that for large $\vartheta$ the concentration at the surface $(\tilde{r}=1)$ becomes fixed at its final value $\left(c=c_{f}\right)$ as uptake or loss take place. In fact, it is often an excellent approximation to replace the boundary condition in Eq. 22 with $\rho_{\mathrm{w}}=\rho_{\mathrm{w}_{f}}$. This 

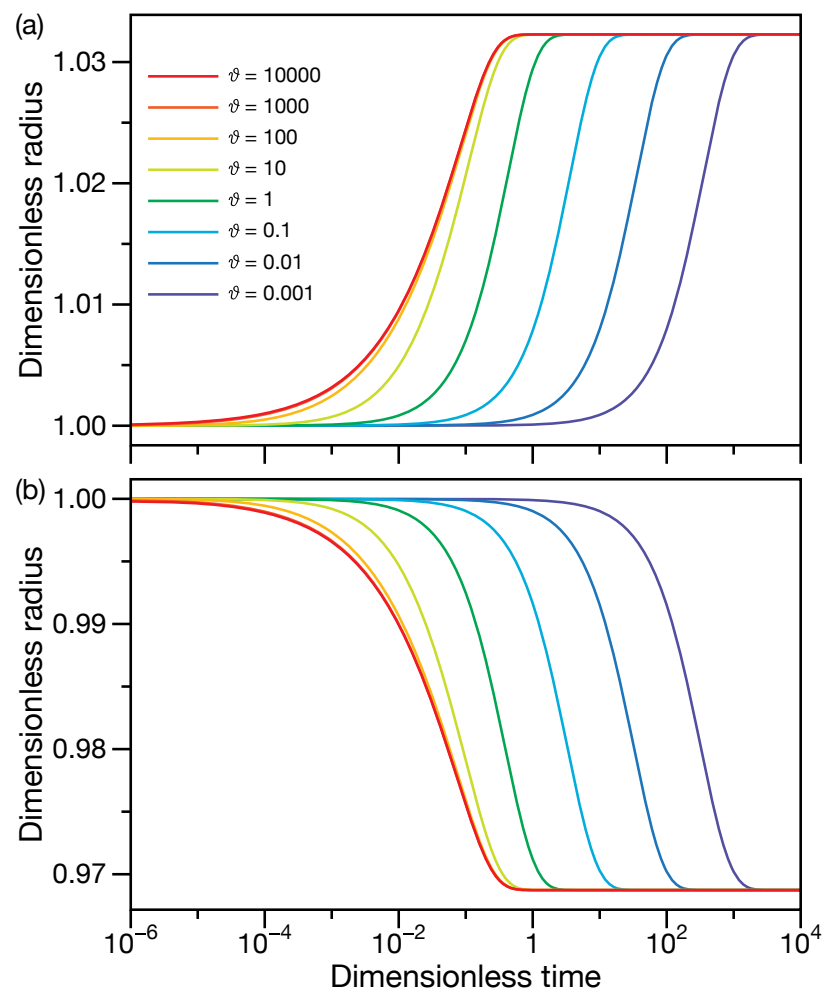

Figure 1: Dimensionless radius as a function of dimensionless time for several different values of $\vartheta$. Curves were calculated using Eq. 30. The dimensionless initial concentration, $c_{i}$, and the dimensionless final concentration, $c_{f}$, were (a) $c_{i}=0.01$ and $c_{f}=0.1$ and $(\mathrm{b}) c_{i}=0.1$ and $c_{f}=0.01$.

condition assumes that the concentration at the particle surface instantaneously reaches its final concentration. It has been used when analyzing isotopic water diffusion experiments ${ }^{29,30}$ as it is satisfactory for a wide range of atmospherically relevant temperatures and $\mathrm{RHs}^{28}$

\section{Evaluating the Time-dependent Radius during Water Uptake or Loss}

In order to apply the expressions derived in Section $3, \bar{D}$ needs to be defined. For a viscous organic aerosol during a stepwise change in $\mathrm{RH}$, diffusivity can vary as a function of concen- 

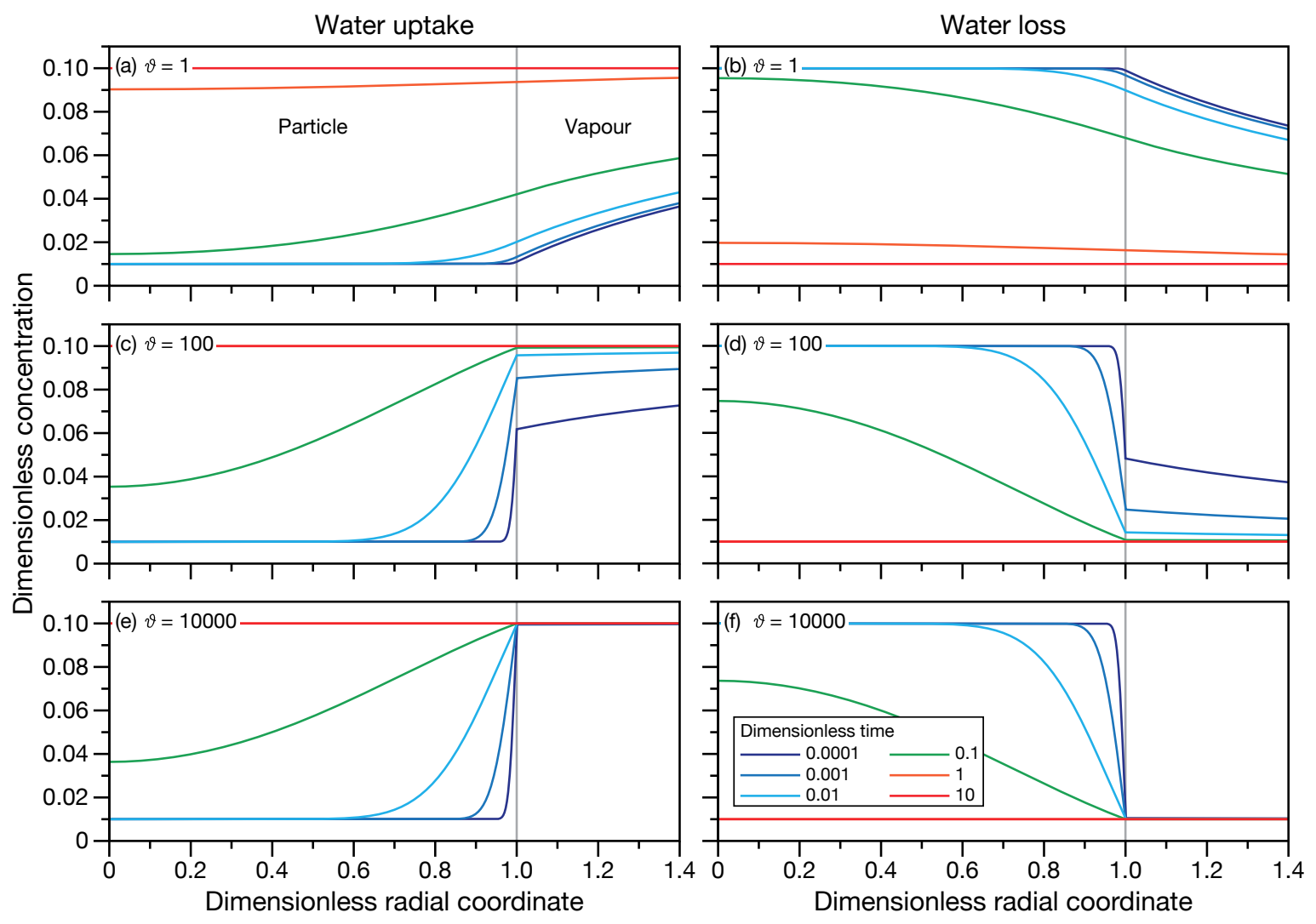

Figure 2: Dimensionless concentration profiles inside a particle and outside its surface during uptake (left panels: a, c, and e) or loss (right panels: b, d, and f) for three different values of $\vartheta$ at various dimensionless times. The dimensionless initial concentration, $c_{i}$, and the dimensionless final concentration, $c_{f}$, were $\left(\mathrm{a}, \mathrm{c}\right.$, and e) $c_{i}=0.1$ and $c_{f}=1$ and $(\mathrm{b}, \mathrm{d}$, and $\mathrm{f}) c_{i}=1$ and $c_{f}=0.1$. Concentration profiles were calculated using Eq. 31 and a dimensionless version of Eq. 4. 
tration by orders of magnitude during water uptake and loss. This is known to lead to a large difference in the timescale of uptake and loss of water when the RH is cycled between two values. $^{7,8,12,31,32}$ During an RH cycle, water uptake will be much faster than water loss. Numerical simulations have shown that this behaviour can be explained with a concentration-dependent diffusivity. ${ }^{12,31}$ Motivated by these observations and calculations, the value of $D_{c}$ that will be used when calculating $\bar{D}$ will be more heavily weighted towards its value at $\rho_{\mathrm{w}_{f}}$. We define $\bar{D}$ as a weighted average

$$
\bar{D}=\frac{\int_{\rho_{\mathrm{w}_{i}}}^{\rho_{\mathrm{w}_{f}}} \phi\left(\rho_{\mathrm{w}}\right) D_{c}\left(\rho_{\mathrm{w}}\right) d \rho_{\mathrm{w}}}{\int_{\rho_{\mathrm{w}_{i}}}^{\rho_{\mathrm{w}_{f}}} \phi\left(\rho_{\mathrm{w}}\right) d \rho_{\mathrm{w}}} .
$$

The weight function, $\phi$, used here is the Heaviside step function, $H\left(x-x_{0}\right)$, where $x=\left(\rho_{\mathrm{w}}-\right.$ $\left.\rho_{\mathrm{w}_{i}}\right) /\left(\rho_{\mathrm{w}_{f}}-\rho_{\mathrm{w}_{i}}\right)$ and $x_{0}$ is set to 0.35 during all calculations. The accuracy of this simple weight function will be subsequently demonstrated.

We now consider three different aqueous systems that have been used as a model system for a wide range of atmospheric organic aerosols: water and either shikimic acid, citric acid, or sucrose. ${ }^{9}$ For instance, aqueous shikimic acid particles have been used to investigate heterogeneous chemistry and mass transport in high viscosity, solid and semi-solid aerosols. ${ }^{28,29,33-36}$ Across a range of atmospherically relevant temperatures and RHs, the diffusivity of the shikimic acid-water system has a very strong concentration dependence. ${ }^{34}$

Fig. 3 shows the time-dependent radius of aqueous organic aerosol particles in response to several different stepwise changes in RH. In all calculations presented in this work for these systems, parameters for aqueous shikimic acid were taken from Ref. 34 and parameters for aqueous sucrose were taken from Ref. 12. The density and activity parameterizations for 

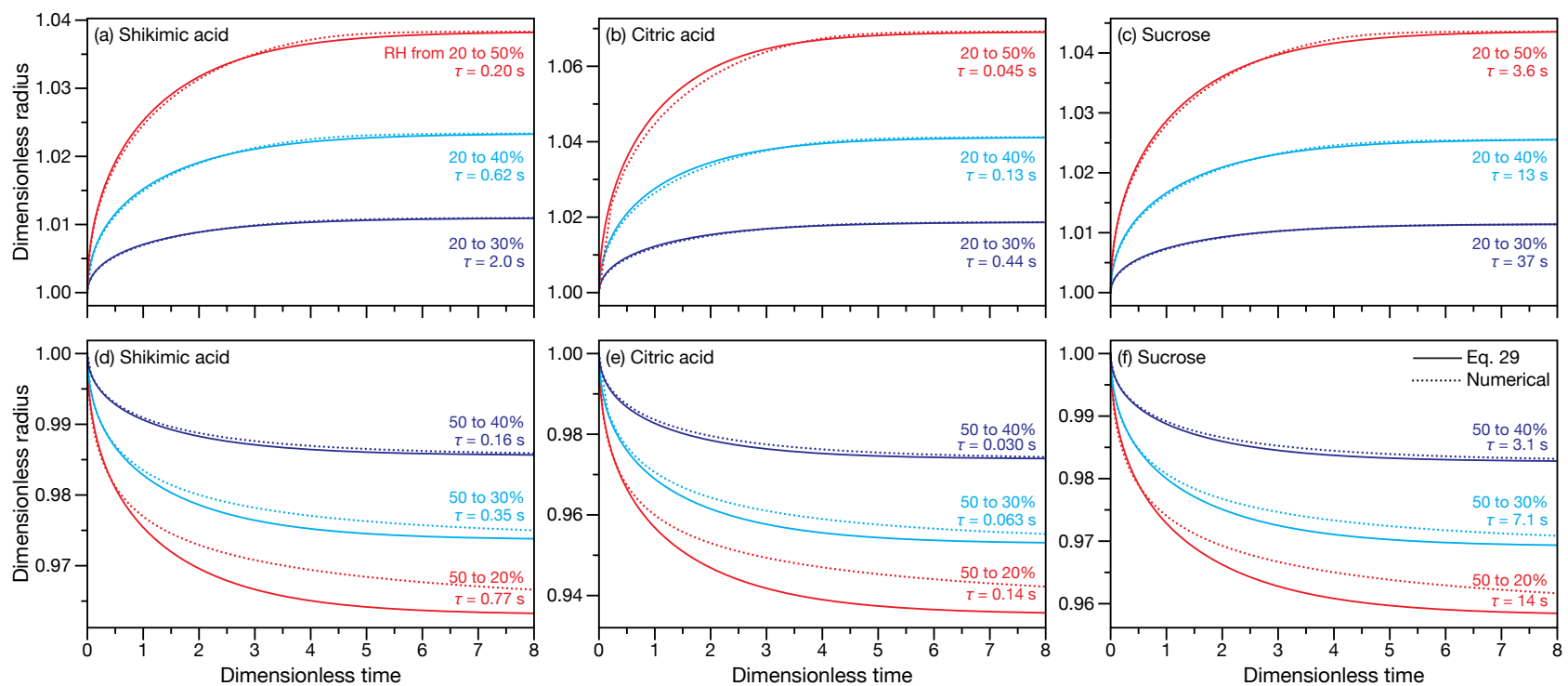

Figure 3: The dimensionless radius, $a(t) / a_{0}$, as a function of dimensionless time, $t / \tau$, for particles consisting of water and either shikimic acid (a and d), citric acid (b and e), or sucrose (c and f) in response to several different stepwise changes in RH calculated using either Eq. 29 (solid lines) or the numerical method from Ref. 12 (dotted lines). The changes in RH occur at $t=0$ and the initial and final values of the $\mathrm{RH}$ are indicated next to their calculated curves. For each pair of RHs, the value of $\tau$ used to non-dimensionalize the time was found using the numerical calculation and is listed in the figure. For all calculations, the initial radius, $a_{0}$, was set to $1 \mu \mathrm{m}$ and $T=298 \mathrm{~K}$.

aqueous citric acid were taken from Ref. 37 and the activity-dependent diffusivity, $D_{\mathrm{CA}}$, was ${ }^{23,29}$

$$
\log _{10} D_{\mathrm{CA}}=b_{1}+b_{2} a_{\mathrm{w}}+b_{3} a_{\mathrm{w}}^{2}+b_{4} a_{\mathrm{w}}^{3},
$$

where $a_{\mathrm{w}}$ is the water activity, $b_{1}=-16.077, b_{2}=16.116, b_{3}=-17.532, b_{4}=8.7575$, and the units of $D_{\mathrm{CA}}$ are $\mathrm{m}^{2} / \mathrm{s}$. Due to the strong dependence of the diffusivity on concentration, the timescale of water uptake and loss can vary tremendously for different stepwise changes in RH. For example, in aqueous shikimic acid at an RH of $20 \%, D=8.38 \times 10^{-15} \mathrm{~m}^{2} / \mathrm{s}$ whereas at an RH of $50 \%, D=9.37 \times 10^{-13} \mathrm{~m}^{2} / \mathrm{s}$ at $T=298 \mathrm{~K}$.

As shown in Fig. 3, during water uptake (a-c), Eq. 29 yields results that are in good 
agreement with the numerical solution. For water loss (e-f), Eq. 29 is similar to the numerical solution for the 50 to $40 \%$ change in $\mathrm{RH}$ but for larger decreases in $\mathrm{RH}$, the correspondence between the two results is less satisfactory. Based on the derivation presented in Sections 2 and 3, there are several approximations that could lead to inaccuracies in the calculated timedependent radius. Here, though, the main source of error between Eq. 29 and the numerical results is the assumption of a constant diffusivity used in the derivation of Eq. 29. The solution to the resulting linear boundary value problem cannot accurately describe the "long-tail" that is seen at longer times in the numerical results. The nonlinear behaviour that dominates at longer times cannot be captured by the linear solution no matter what value of $\bar{D}$ is chosen. This discrepancy makes the results that will be discussed in Section 5 all the more surprising: the characteristic time for these cases can be accurately calculated using the linear solution.

In addition to our consideration of water uptake and loss by organic aerosols, we can also examine the RH-dependent change in size of an aqueous inorganic aerosol particle due to water uptake and loss using Eq. 29. Here we consider a particle composed of water and ammonium sulfate. The activity and density of aqueous ammonium sulfate are well-characterized, ${ }^{38}$ however measurements of the concentration-dependent diffusivity are limited to a smaller range of concentrations. ${ }^{39}$ Using available data, the diffusivity, $D_{\mathrm{AS}}$, was fitted as a function of the mass fraction of ammonium sulfate, $w$, to give

$$
D_{\mathrm{AS}}=d_{1}+d_{2} w^{1 / 2}+d_{3} w^{1 / 3}+d_{4} w^{1 / 4}
$$

where $d_{1}=1.531 \times 10^{-9}, d_{2}=5.751 \times 10^{-10}, d_{3}=-4.006 \times 10^{-10}, d_{4}=-9.042 \times 10^{-10}$, and the units of $D_{\text {AS }}$ are $\mathrm{m}^{2} / \mathrm{s}$.

Fig. 4 shows the time-dependent radius of an aqueous ammonium sulfate particle in response 
to several different stepwise changes in $\mathrm{RH}$. In all cases, at $t=0$ the $\mathrm{RH}$ is changed from its initial value to its final value. For small changes in $\mathrm{RH}$, it can be seen that Eq. 29 closely matches the numerical results. For larger changes in $\mathrm{RH}$, the differences between the numerical and analytic results increase although the accuracy is still reasonably good. The main reason for the good agreement is that, in the regions across which the $\mathrm{RH}$ is being changed for the calculations in Fig. 4, the diffusivity is approximately constant. This is in contrast to the aqueous organic examples considered in Fig. 3 where, in some cases, the diffusivity changed by orders of magnitude across the $\mathrm{RH}$ range present in the stepwise changes.

\section{The Characteristic Equilibration Time of an Aqueous Aerosol Particle}

For a stepwise change in $\mathrm{RH}$ at $t=0$, the total amount of water at the characteristic equilibration time, $Q_{\mathrm{w}}(\tau)$, is defined as

$$
Q_{\mathrm{w}}(\tau)=\left(Q_{\mathrm{w}_{i}}-Q_{\mathrm{w}_{f}}\right) / e+Q_{\mathrm{w}_{f}},
$$

during either water uptake or loss. In this expression, $Q_{\mathrm{w}_{i}}$ is the total amount of water in the particle prior to the stepwise change in $\mathrm{RH}$ and $Q_{\mathrm{w}_{f}}$ is the total amount of water in the particle as $t \rightarrow \infty$.

Using results from Sections 2 and 3, it is useful for our subsequent discussion to define the dimensionless amount of water as

$$
\tilde{Q}_{\mathrm{w}}=c_{f}+6 \vartheta^{2}\left(c_{i}-c_{f}\right) \sum_{n=1}^{\infty} \frac{B_{n}}{\lambda_{n}^{2}} \sin \lambda_{n} e^{-\lambda_{n}^{2} \tilde{t}}
$$

where $\tilde{Q}_{\mathrm{w}}=3 Q_{\mathrm{w}}(t) / 4 \pi \epsilon a_{0}^{3}$. 

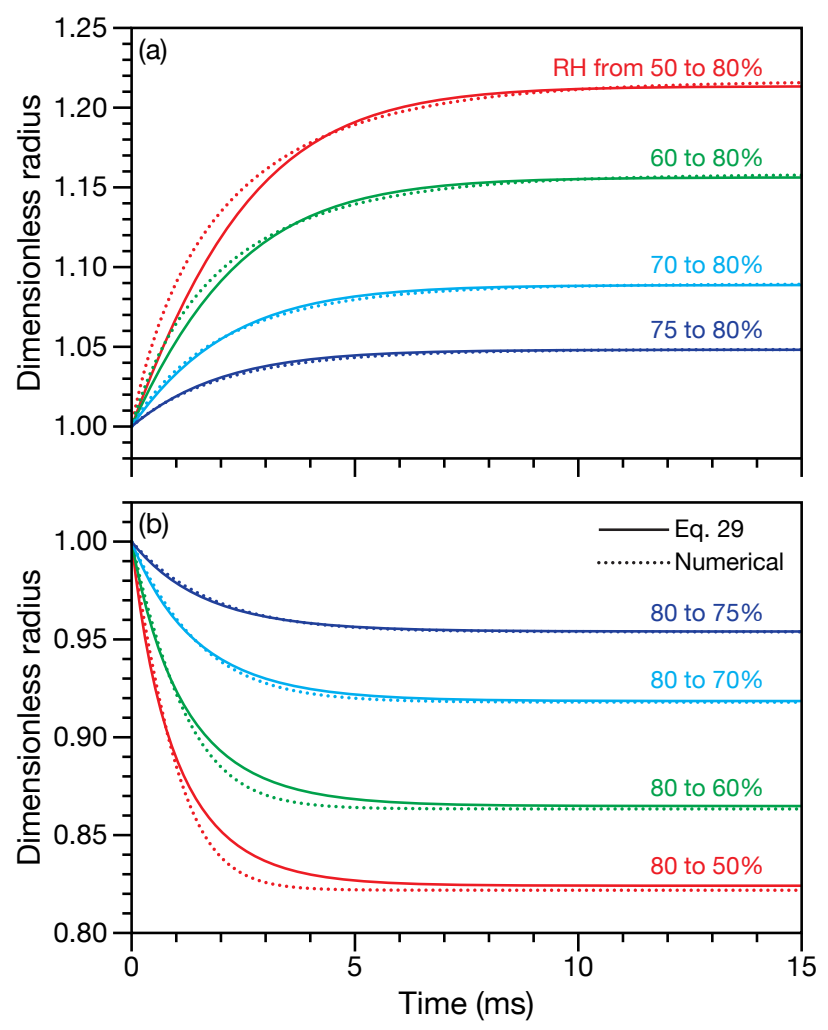

Figure 4: The time-dependent dimensionless radius, $a(t) / a_{0}$, for an aqueous ammonium sulfate particle in response to several different stepwise changes in RH calculated using either Eq. 29 (solid lines) or the numerical method from Ref. 12 (dotted lines). The changes in RH occur at $t=0$ and the initial and final values of the $\mathrm{RH}$ are indicated next to their calculated curves. For all calculations, the initial radius, $a_{0}$, was set to $1 \mu \mathrm{m}$ and $T=298 \mathrm{~K}$. 
Despite the definition in Eq. 35, it should not be assumed that the uptake and loss of water are first-order processes. ${ }^{40}$ Consequently, calculating the characteristic equilibration time (or $e$-folding time), $\tau$, using numerical methods can be a time consuming process as the simulation needs to be run until $Q_{\mathrm{w}}(\tau)$ is reached in order to accurately determine $\tau$. Therefore, a simple expression for the rapid calculation of $\tau$ is very desirable. Examining the $n=1$ term in Eq. 36 and recognizing that $\lambda_{1}$ approaches $\pi$ as $\vartheta$ becomes much greater than one, we arrive at the simple definition of $\tau^{*}=a_{0}^{2} / \pi^{2} \bar{D}$. However, evaluation of Eq. 36 showed that $\tau$ was always approximately equal to $0.55 \tau^{*}$ when $\vartheta \gg 1$. This is shown in Fig. 5. Therefore, a suitable expression for the characteristic equilibration time of viscous aqueous aerosol particles should be

$$
\tau=0.55 \frac{a_{0}^{2}}{\pi^{2} \bar{D}}
$$

Figs. 6 to 8 show a comparison between $\tau$ calculated using a numerical method ${ }^{12}$ and $\tau$ calculated using Eq. 37 for the model systems of (a) aqueous shikimic acid, (b) aqueous citric acid, and (c) aqueous sucrose. In all calculations, $a_{0}=1 \mu \mathrm{m}$. The range of initial and final RHs considered is 0 to $99 \%$ (the binary particle has no equilibrium at $100 \% \mathrm{RH}$ ). For viscous aerosol particles, equilibration with the surrounding $\mathrm{RH}$ can vary from seconds to years for typical particle sizes and atmospheric conditions. ${ }^{9}$ In Figs. 6 to 8, where the temperature and initial radius are fixed, $\tau$ still shows a variation of up to six orders of magnitude across the $\mathrm{RH}$ range. Overall, Eq. 37 yields excellent agreement with the numerical calculation for $\tau>10^{-1} \mathrm{~s}$ for all three systems. In this regime, Eq. 37 also correctly predicts that the $\tau=0$ line that occurs when the initial $\mathrm{RH}$ is equal to the final $\mathrm{RH}$ is not a line of reflection for the contour lines of $\tau$. The function $\tau$ is not symmetric for all pairs of RHs. Specifically, for two given RHs, $\tau$ is always greater when the lower $\mathrm{RH}$ value is used as the final $\mathrm{RH}$. In contrast, a naive 

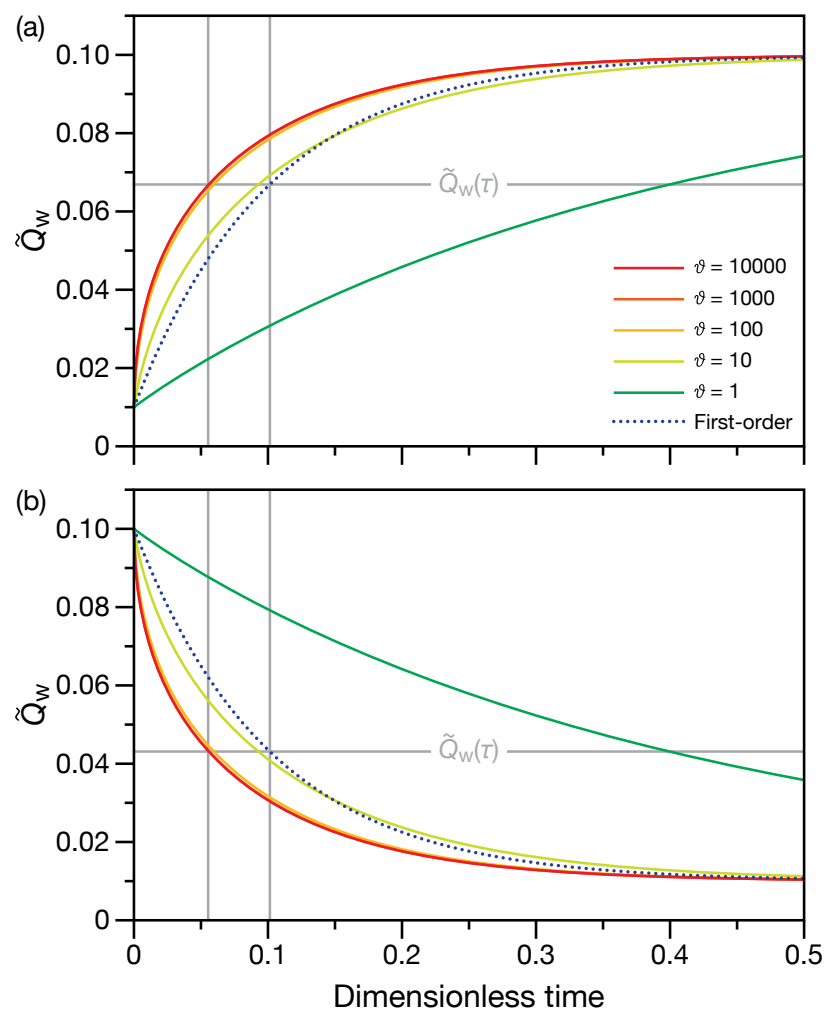

Figure 5: The dimensionless total amount of water, $\tilde{Q}_{\mathrm{w}}$, in a particle as a function of dimensionless time for several different values of $\vartheta$. Curves were calculated using Eq. 36. The dimensionless initial concentration, $c_{i}$, and the dimensionless final concentration, $c_{f}$, were (a) $c_{i}=0.01$ and $c_{f}=0.1$ and (b) $c_{i}=0.1$ and $c_{f}=0.01$. First-order (a) uptake and (b) loss is also shown. The line indicated by $\tilde{Q}_{\mathrm{w}}(\tau)$ intersects the first-order curve at $\tilde{t}=0.101$ and the curves with large values of $\vartheta(1000$ and 10000$)$ at $\tilde{t}=0.0558$. The ratio of these two dimensionless times leads to the choice of $\tau=0.55 \tau^{*}$ in Section 5 . 


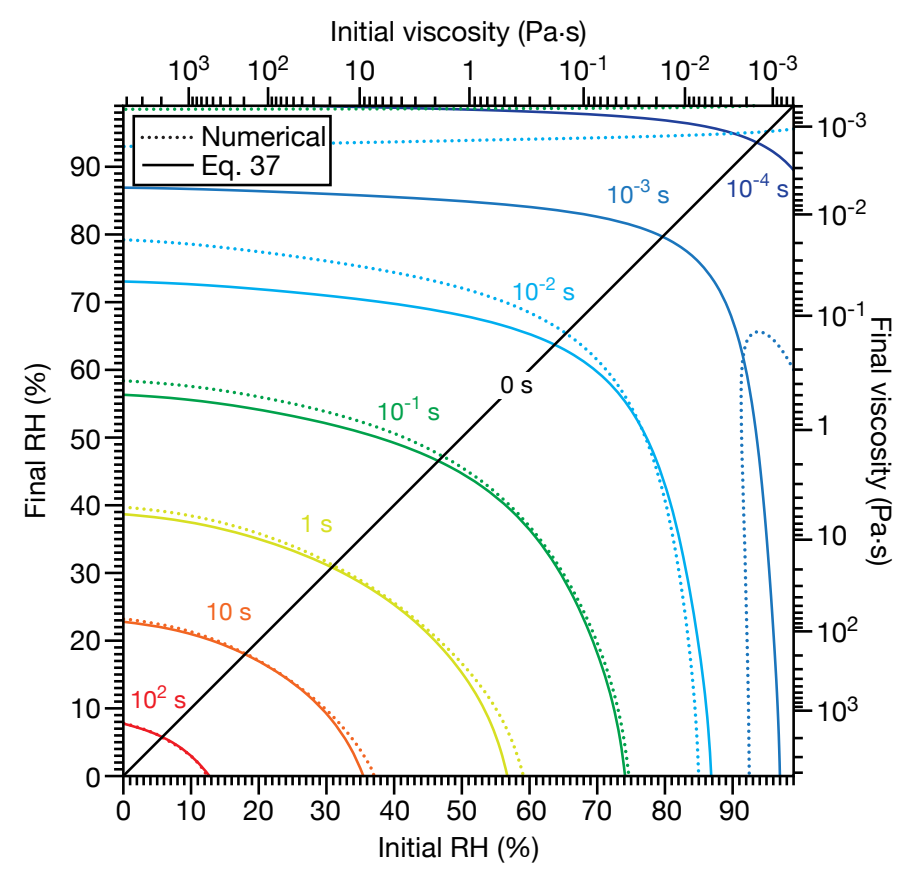

Figure 6: Characteristic equilibration time, $\tau$, for an aqueous shikimic acid aerosol particle for various stepwise changes in RH. For all calculations, the initial radius, $a_{0}$, was set to $1 \mu \mathrm{m}$ and $T=298 \mathrm{~K}$. Dynamic viscosity was calculated with the Stokes-Einstein equation where the molecular radius of water was $0.2 \mathrm{~nm}$. The $\tau=0$ line is added to guide the eye.

expression for the lifetime, e.g. $a_{0}^{2} / \pi^{2} D_{m}$, where $D_{m}$ is simply the diffusivity at a midpoint between the initial and final $\mathrm{RH}$, would not only be inaccurate but it would also incorrectly predict the $\tau=0$ to be a line of reflection. At high initial or final RHs, i.e. when $\tau<10^{-1} \mathrm{~s}$, the agreement between the two calculations is poor. This is because the assumption that $\vartheta \gg 1$ that we relied on in our derivation of Eq. 37 is no longer valid and that equation becomes very inaccurate.

In order to understand the phase states over which Eq. 37 is applicable we apply the StokesEinstein equation to relate diffusivity to viscosity. Setting the molecular radius of water to $0.2 \mathrm{~nm},{ }^{5}$ we can calculate the dynamic viscosity of the particle when it is in equilibrium with the surrounding RH. These viscosities are shown as axes in Figs. 6 to 8 as initial and final 


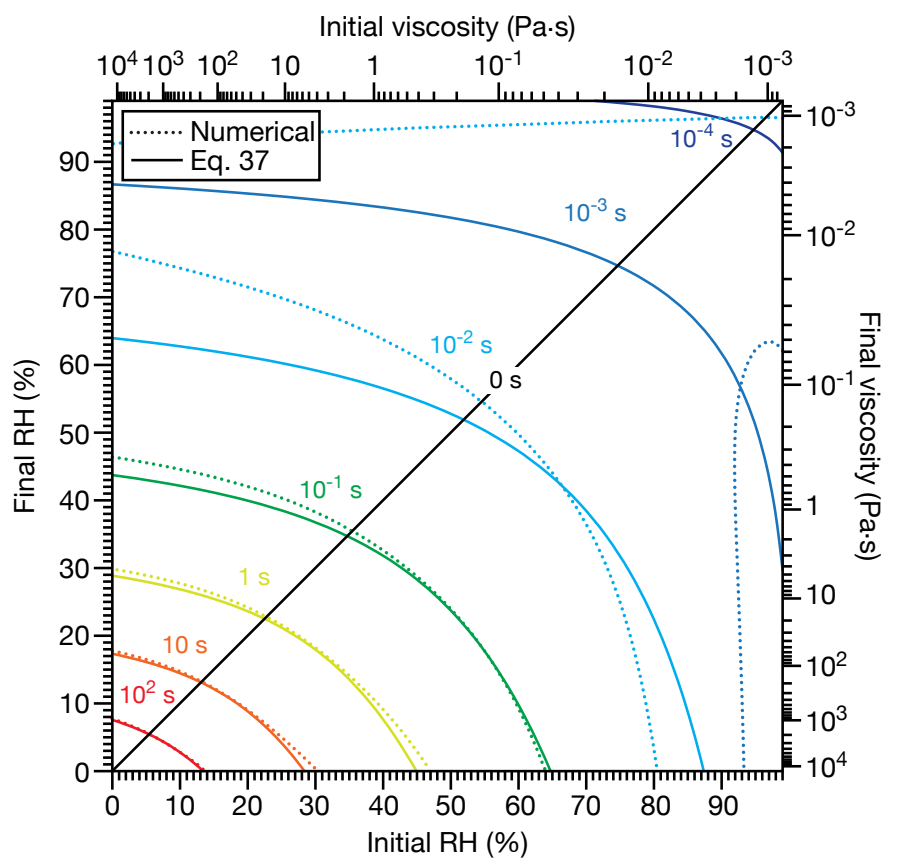

Figure 7: Characteristic equilibration time, $\tau$, for an aqueous citric acid aerosol particle for various stepwise changes in $\mathrm{RH}$. For all calculations, the initial radius, $a_{0}$, was set to $1 \mu \mathrm{m}$ and $T=298 \mathrm{~K}$. Dynamic viscosity was calculated with the Stokes-Einstein equation where the molecular radius of water was $0.2 \mathrm{~nm}$. The $\tau=0$ line is added to guide the eye. 


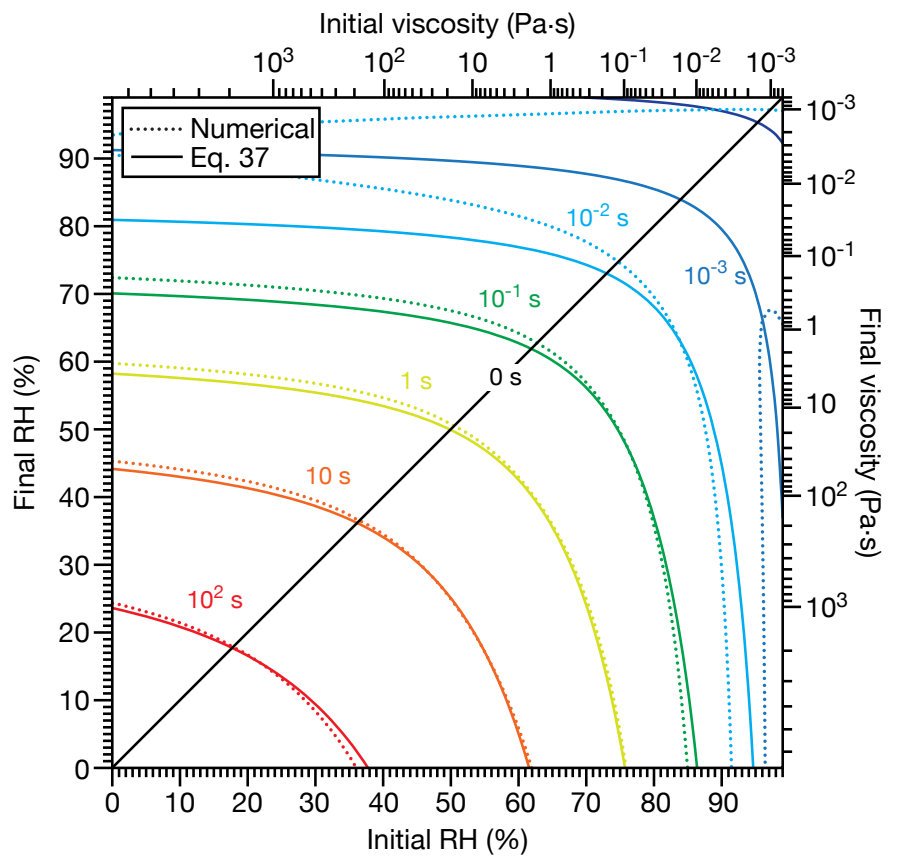

Figure 8: Characteristic equilibration time, $\tau$, for an aqueous sucrose aerosol particle for various stepwise changes in RH. For all calculations, the initial radius, $a_{0}$, was set to $1 \mu \mathrm{m}$ and $T=$ 298 K. Dynamic viscosity was calculated with the Stokes-Einstein equation where the molecular radius of water was $0.2 \mathrm{~nm}$. The $\tau=0$ line is added to guide the eye. 
viscosities. A dynamic viscosity of around $10^{2} \mathrm{~Pa} \cdot \mathrm{s}$ is commonly used as the transition from the liquid to semi-solid phase state. ${ }^{41,42}$ It can be seen that, during water uptake and loss, Eq. 37 will be accurate so long as the particle remains in a phase state whose viscosity is that of a semi-solid or greater (e.g. it will also produce accurate results for solid glassy particles). In fact, Eq. 37 produces results that are accurate down to $1 \mathrm{~Pa} \cdot \mathrm{s}$, so it can be applied to high viscosity liquids as well.

\section{Conclusion}

We have derived expressions for (i) the time-dependent radius and (ii) the characteristic equilibration time of a binary aqueous particle in response to a stepwise change in the surrounding RH. The derivation illustrates the role that both convection and diffusion play in water transport in a particle and the associated increase or decrease in size that occurs during a stepwise change in RH. Specifically, the diffusion of water in the particle induced convection by changing the density of the aqueous medium. These density changes led to either swelling or shrinkage and allowed us to derive an expression for the time-dependent radius of the particle. Evaluation of the equations demonstrated that heavily weighting the diffusivity towards its value at the final $\mathrm{RH}$ produced accurate results. This was applied to aqueous systems that are considered to be models for high viscosity aerosol particles. Even though diffusivity can vary by several orders of magnitude during water uptake or loss, the calculated characteristic equilibration time during a change in RH was in excellent agreement with the numerical solution for semi-solid and solid phases as well as liquids whose viscosity is above $1 \mathrm{~Pa} \cdot \mathrm{s}$. As it is known that the characteristic equilibration time is not a symmetric function of the initial and final RH states, it was expected that weighting the diffusivity more heavily towards its final state would improve 
the accuracy of the calculation. What was surprising, though, was that a simple weighting function could yield such a close match to numerical calculations and maintain this accuracy over such a wide range of initial and final conditions. This illustrates the importance of the final state during water uptake and loss and, of course, provides an expression that can be used to rapidly calculate the characteristic equilibration time.

\section{Acknowledgements}

T.C.P. acknowledges support from the Natural Sciences and Engineering Research Council of Canada (NSERC).

\section{References}

1. Philip, J. R. Kinetics of growth and evaporation of droplets and ice crystals. J. Atmos. Sci. 1965, 22, 196-206.

2. Vesala, T.; Kulmala, M.; Rudolf, R.; Vrtala, A.; Wagner, P. E. Models for condensational growth and evaporation of binary aerosol particles. J. Aerosol Sci. 1997, 28, 565-598.

3. Widmann, J. F.; Davis, E. J. Evaporation of multicomponent droplets. Aerosol Sci. Technol. 1997, 27, 243-254.

4. Mai, H.; Shiraiwa, M.; Flagan, R. C.; Seinfeld, J. H. Under what conditions can equilibrium gas-particle partitioning be expected to hold in the atmosphere? Environ. Sci. Technol. 2015, 49, 11485-11491.

5. Bones, D. L.; Reid, J. P.; Lienhard, D.; Krieger, U. K. Comparing the mechanism of water 
condensation and evaporation in glassy aerosol. Proc. Natl. Acad. Sci. USA 2012, 109, $11613-11618$.

6. Berkemeier, T.; Shiraiwa, M.; Pöschl, U.; Koop, T. Competition between water uptake and ice nucleation by glassy organic aerosol particles. Atmos. Chem. Phys. 2014, 14, $12513-12531$.

7. Rickards, A. M. J.; Song, Y.-C.; Miles, R. E. H.; Preston, T. C.; Reid, J. Uncertainties and variabilities in measurements of water transport in viscous aerosol. Phys. Chem. Chem. Phys. 2015, 17, 10059-10073.

8. Price, H. C.; Mattsson, J.; Zhang, Y.; Bertram, A. K.; Davies, J. F.; Grayson, J. W.; Martin, S. T.; O'Sullivan, D.; Reid, J. P.; Rickards, A. M. J.; et al. Water diffusion in atmospherically relevant $\alpha$-pinene secondary organic material. Chem. Sci. 2015, 6, 48764883.

9. Lienhard, D. M.; Huisman, A. J.; Krieger, U. K.; Rudich, Y.; Marcolli, C.; Luo, B. P.; Bones, D. L.; Reid, J. P.; Lambe, A. T.; Canagaratna, M. R.; et al. Viscous organic aerosol particles in the upper troposphere: diffusivity-controlled water uptake and ice nucleation? Atmos. Chem. Phys. 2015, 15, 13599-13613.

10. Shiraiwa, M.; Li, Y.; Tsimpidi, A. P.; Karydis, V. A.; Berkemeier, T.; Pandis, S. N.; Lelieveld, J.; Koop, T.; Pöschl, U. Global distribution of particle phase state in atmospheric secondary organic aerosols. Nat. Commun. 2017, 8, 1-7.

11. Ingram, S.; Cai, C.; Song, Y.-C.; Glowacki, D. R.; Topping, D. O.; O’Meara, S.; Reid, J. P. Characterising the evaporation kinetics of water and semi-volatile organic compounds 
from viscous multicomponent organic aerosol particles. Phys. Chem. Chem. Phys. 2017, 19, 31634-31646.

12. Zobrist, B.; Soonsin, V.; Luo, B. P.; Krieger, U. K.; Marcolli, C.; Peter, T.; Koop, T. Ultra-slow water diffusion in aqueous sucrose glasses. Phys. Chem. Chem. Phys. 2011, 13, $3514-3526$.

13. Shiraiwa, M.; Pfrang, C.; Koop, T.; Pöschl, U. Kinetic multi-layer model of gas-particle interactions in aerosols and clouds (km-gap): linking condensation, evaporation and chemical reactions of organics, oxidants and water. Atmos. Chem. Phys. 2012, 12, 2777-2794.

14. O’Meara, S.; Topping, D. O.; McFiggans, G. The rate of equilibration of viscous aerosol particles. Atmos. Chem. Phys. 2016, 16, 5299-5313.

15. O’Meara, S.; Topping, D. O.; Zaveri, R. A.; McFiggans, G. An efficient approach for treating composition-dependent diffusion within organic particles. Atmos. Chem. Phys. 2017, 17, 10477-10494.

16. Zieger, P.; Fierz-Schmidhauser, R.; Gysel, M.; Ström, J.; Henne, S.; Yttri, K. E.; Baltensperger, U.; Weingartner, E. Effects of relative humidity on aerosol light scattering in the Arctic. Atmos. Chem. Phys. Discuss. 2010, 10, 3659-3698.

17. Tong, H.-J.; Reid, J. P.; Bones, D. L.; Luo, B. P.; Krieger, U. K. Measurements of the timescales for the mass transfer of water in glassy aerosol at low relative humidity and ambient temperature. Atmos. Chem. Phys. 2011, 11, 4739-4754.

18. Wang, B.; Lambe, A. T.; Massoli, P.; Onasch, T. B.; Davidovits, P.; Worsnop, D. R.; Knopf, D. A. The deposition ice nucleation and immersion freezing potential of amorphous 
secondary organic aerosol: Pathways for ice and mixed-phase cloud formation. J. Geophys. Res. Atmos. 2012, 117, D16209.

19. Renbaum-Wolff, L.; Grayson, J. W.; Bateman, A. P.; Kuwata, M.; Sellier, M.; Murray, B. J.; Shilling, J. E.; Martin, S. T.; Bertram, A. K. Viscosity of $\alpha$-pinene secondary organic material and implications for particle growth and reactivity. Proc. Natl. Acad. Sci. USA 2013, 110, 8014-8019.

20. Knopf, D. A.; Alpert, P. A.; Wang, B. The role of organic aerosol in atmospheric ice nucleation: a review. ACS Earth Space Chem. 2018, 2, 168-202.

21. Liu, P.; Song, M.; Zhao, T.; Gunthe, S. S.; Ham, S.; He, Y.; Qin, Y. M.; Gong, Z.; Amorim, J. C.; Bertram, A. K.; et al. Resolving the mechanisms of hygroscopic growth and cloud condensation nuclei activity for organic particulate matter. Nat. Commun. 2018, 1-10.

22. Alsoy, S.; Duda, J. L. Influence of swelling and diffusion-induced convection on polymer sorption processes. AIChE J. 2002, 9, 1849-1855.

23. Preston, T. C.; Davies, J. F.; Wilson, K. R. The frequency-dependent response of single aerosol particles to vapour phase oscillations and its application in measuring diffusion coefficients . Phys. Chem. Chem. Phys. 2017, 19, 3922-3931.

24. Tagantsev, D. K.; Ivanenko, D. V. Validity of the second fick's law for modeling ionexchange diffusionin non-crystalline viscoelastic media. J. Appl. Phys. 2016, 119, 134905.

25. Schweiger, G. In-situ determination of the molecular composition of aerosol particles in a monodisperse model aerosol. Part. Part. Syst. Char. 1987, 4, 67-73. 
26. Schweiger, G. Raman scattering on microparticles: size dependence. J. Opt. Soc. Am. B 1991, 8, 1770-1778.

27. Schweiger, G.; Lange, S. Structural resonances in the total Raman- and fluorescencescattering cross section: concentration-profile dependence. J. Opt. Soc. Am. B 1996, 13, $1864-1872$.

28. Moridnejad, A.; Preston, T. C. Models of isotopic water diffusion in spherical aerosol particles. J. Phys. Chem. A 2016, 120, 9759-9766.

29. Davies, J. F.; Wilson, K. R. Raman spectroscopy of isotopic water diffusion in ultraviscous, glassy, and gel states in aerosol by use of optical tweezers. Anal. Chem. 2016, 88, 23612366.

30. Price, H. C.; Murray, B. J.; Mattsson, J.; O’Sullivan, D.; Wilson, T. W.; Baustian, K. J.; Benning, L. G. Quantifying water diffusion in high-viscosity and glassy aqueous solutions using a Raman isotope tracer method. Atmos. Chem. Phys. 2014, 14, 3817-3830.

31. Lienhard, D. M.; Huisman, A. J.; Bones, D. L.; Te, Y.-F.; Luo, B. P.; Krieger, U. K.; Reid, J. P. Retrieving the translational diffusion coefficient of water from experiments on single levitated aerosol droplets. Phys. Chem. Chem. Phys. 2014, 16, 16677-16683.

32. Lu, J. W.; Rickards, A. M. J.; Walker, J. S.; Knox, K. J.; Miles, R. E. H.; Reid, J. P.; Signorell, R. Timescales of water transport in viscous aerosol: measurements on submicron particles and dependence on conditioning history. Phys. Chem. Chem. Phys. 2014, 16, 9819-9830. 
33. Steimer, S. S.; Berkemeier, T.; Gilgen, A.; Krieger, U. K.; Peter, T.; Shiraiwa, M.; Ammann, M. Shikimic acid ozonolysis kinetics of the transition from liquid aqueous solution to highly viscous glass. Phys. Chem. Chem. Phys. 2015, 17, 31101-31109.

34. Steimer, S. S.; Krieger, U. K.; Te, Y. F.; Lienhard, D. M.; Huisman, A. J.; Luo, B. P.; Ammann, M.; Peter, T. Electrodynamic balance measurements of thermodynamic, kinetic, and optical aerosol properties inaccessible to bulk methods. Atmos. Meas. Tech. 2015, 8, $2397-2408$.

35. Berkemeier, T.; Steimer, S.; Krieger, U. K.; Peter, T.; Pöschl, U.; Ammann, M.; Shiraiwa, M. Ozone uptake on glassy, semi-solid and liquid organic matter and the role of reactive oxygen intermediates in atmospheric aerosol chemistry. Phys. Chem. Chem. Phys. 2016, $18,12662-12674$.

36. Bastelberger, S.; Krieger, U. K.; Luo, B. P.; Peter, T. Time evolution of steep diffusion fronts in highly viscous aerosol particles measured with Mie resonance spectroscopy. $J$. Chem. Phys. 2018, 149, 244506.

37. Lienhard, D. M.; Bones, D. L.; Zuend, A.; Krieger, U. K.; Reid, J. P.; Peter, T. Measurements of thermodynamic and optical properties of selected aqueous organic and organic-inorganic mixtures of atmospheric relevance. J. Phys. Chem. A 2012, 116, 99549968.

38. Tang, I. N.; Munkelwitz, H. R. Water activities, densities, and refractive indices of aqueous sulfates and sodium nitrate droplets of atmospheric importance. J. Geophys. Res. 1994, 99, 18801-18808. 
39. Leaist, D. G.; Hao, L. Binary mutual diffusion coefficients of aqueous ammonium and potassium sulfates at $25^{\circ} \mathrm{C}$. J. Solution Chem. 1992, 21, 345-350.

40. Moridnejad, A.; Preston, T. C.; Krieger, U. K. Tracking water sorption in glassy aerosol particles using morphology-dependent resonances. J. Phys. Chem. A 2017, 121, 8176-8184.

41. Shiraiwa, M.; Ammann, M.; Koop, T.; Pöschl, U. Gas uptake and chemical aging of semisolid organic aerosol particles. Proc. Natl. Acad. Sci. USA 2011, 108, 11003-11008.

42. Reid, J. P.; Bertram, A. K.; Topping, D. O.; Laskin, A.; Martin, S. T.; Petters, M. D.; Pope, F. D.; Rovelli, G. The viscosity of atmospherically relevant organic particles. Nat. Commun. 2018, 9, 1-14. 
Abstract Graphic

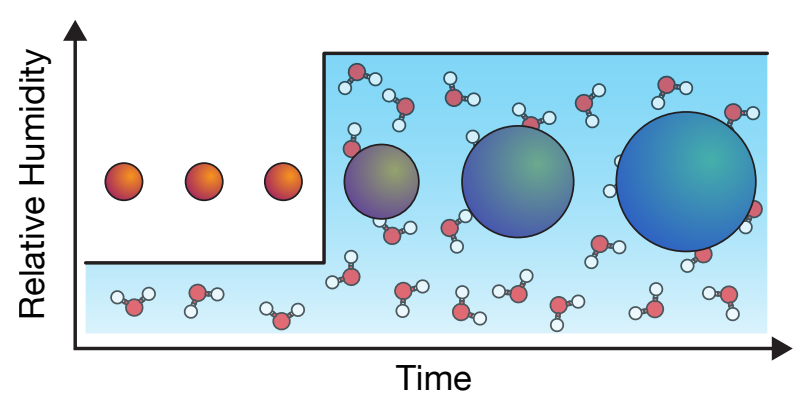

